\title{
Non-canonical activation of hedgehog in prostate cancer cells mediated by the interaction of transcriptionally active androgen receptor proteins with Gli3
}

\author{
$\mathrm{Na} \mathrm{Li}{ }^{1} \cdot$ Sarah Truong ${ }^{1,2} \cdot$ Mannan Nouri $^{2} \cdot$ Jackson Moore ${ }^{1} \cdot$ Nader Al Nakouzi $^{1} \cdot$ Amy Anne Lubik $^{1} \cdot$ Ralph Buttyan $^{2,3}$
}

Received: 19 July 2017 / Revised: 18 October 2017 / Accepted: 29 November 2017 / Published online: 12 February 2018

(c) The Author(s) 2018. This article is published with open access

\begin{abstract}
Hedgehog $(\mathrm{Hh})$ is an oncogenic signaling pathway that regulates the activity of Gli transcription factors. Canonical $\mathrm{Hh}$ is a Smoothened- (Smo-) driven process that alters the post-translational processing of Gli2/Gli3 proteins. Though evidence supports a role for Gli action in prostate cancer $(\mathrm{PCa})$ cell growth and progression, there is little indication that Smo is involved. Here we describe a non-canonical means for activation of Gli transcription in PCa cells mediated by the binding of transcriptionally-active androgen receptors (ARs) to Gli3. Androgens stimulated reporter expression from a Gli-dependent promoter in a variety of AR + PCa cells and this activity was suppressed by an anti-androgen, Enz, or by AR knockdown. Androgens also upregulated expression of endogenous Gli-dependent genes. This activity was associated with increased intranuclear binding of Gli3 to AR that was antagonized by Enz. Fine mapping of the AR binding domain on Gli2 showed that AR recognizes the Gli protein processing domain (PPD) in the C-terminus. Mutations in the arginine-/serine repeat elements of the Gli2 PPD involved in phosphorylation and ubiquitinylation blocked the binding to AR. $\beta$-TrCP, a ubiquitin ligase that recognizes the Gli PPD, competed with AR for binding to this site. AR binding to Gli3 suppressed its proteolytic processing to the Gli3 repressor form (Gli3R) whereas AR knockdown increased Gli3R. Both full-length and truncated ARs were able to activate Gli transcription. Finally, we found that an ARbinding decoy polypeptide derived from the Gli2 Cterminus can compete with Gli3 for binding to AR. Exogenous overexpression of this decoy suppressed Gli transcriptional activity in PCa cells. Collectively, this work identifies a novel pathway for non-canonical activation of Hh signaling in PCa cells and identifies a means for interference that may have clinical relevance for PCa patients.
\end{abstract}

\section{Introduction}

Hedgehog $(\mathrm{Hh})$ is a cell signaling pathway that regulates complex morphogenic processes during embryonic development [1]. Its actions are needed for the development of

$\mathrm{Na} \mathrm{Li}$ and Sarah Truong were equally responsible for the work.

Electronic supplementary material The online version of this article (https://doi.org/10.1038/s41388-017-0098-7) contains supplementary material, which is available to authorized users.

Ralph Buttyan

rbuttyan@prostatecentre.com

The Vancouver Prostate Centre, Vancouver, Canada

2 The Interdisciplinary Oncology Program of The University of British Columbia, Vancouver, Canada

3 Urologic Sciences, The University of British Columbia, Vancouver, Canada the central nervous system [2,3], cranial-facial structures [4], limbs/peripheral appendages [5] and steroidogenic tissues [6], amongst others. Canonical Hh signaling functions to activate cellular transcription from the Gli family of transcription factors (Gli1, Gli2, and Gli3) [7, 8]. Gli proteins recognize a shared concensus DNA binding element, 5'-G-A-C-C-A-C-C-C-A-3' [9], and other permutations of [10]. Classical canonical signaling is initiated by secreted peptides, referred to as hedgehogs, that recognize a cell surface receptor protein, Patched, on target cells [11, 12]. Ligand binding initiates a de-repression of Smoothened (Smo) and, once activated, Smo alters the phosphorylation and proteolytic degradation patterns of Gli2 and Gli3 leading to their transformation from a repressor state to a transcriptional activator state. In turn, transcriptionally active Gli2 and Gli3 induces expression of Gli1 that further augments the active Gli transcriptional state $[13,14]$.

$\mathrm{Hh}$ is also a proliferative stimulus [15] and hyperactive Hh in adult tissues can be oncogenic [16-18]. Basal cell 
carcinoma (BCC) and medulloblastoma (MB), in particular, are sometimes driven by activating or inactivating mutations in upstream $\mathrm{Hh}$ signaling components that enable constitutive Gli transcription $[19,20]$. Other solid tumors, including pancreatic, lung, breast, and prostate cancer $(\mathrm{PCa})$ are also thought to be influenced by $\mathrm{Hh}$, though the types of mutations found in $\mathrm{BCC}$ or $\mathrm{MB}$ are not detected with any significant frequency in these latter tumors. Crosstalk from other oncogenic signaling pathways (MAPK/ras, PI-3 kinase/AKT, TGF- $\beta$, and others), however, may provide a means for non-classical activation of Gli [21-23] in some tumors. Here we show that there is a novel non-canonical pathway for activation of $\mathrm{Hh}$ in $\mathrm{PCa}$ cells that is mechanistically driven by the direct binding of transcriptionally active androgen receptor (AR) proteins to Gli proteins.

$\mathrm{PCa}$ develops and progresses under the influence of androgenic steroids [24]. This influence identifies the importance of the AR protein as a critical effector of $\mathrm{PCa}$ cell survival and growth [25]. Indeed, AR pathway inhibitors provide the foundation for contemporary treatment of metastatic PCa. Unfortunately, hormonal therapeutics used in advanced PCa patients are only palliative and patients eventually recur with disease that is resistant to androgen deprivation (castration resistant prostate cancer or CRPC) as well as the potent anti-androgens now used to treat CRPC. For the most part, however, resistant CRPC cells continue to express AR and remain addicted to it [26]. In these tumors, AR continues to signal to survival and growth endpoints despite the lack of hormonal stimulation. Mechanistically, AR hyperactivity in CRPC has been linked to acquired intratumoral steroidogenesis, overexpression of full length (AR-FL) or C-terminally truncated ARs (t-ARs) or overexpression of AR co-activator proteins [27, 28]. Hh pathway activity may effect more than one of these AR aberrations.

Indeed, we have already shown that a paracrine $\mathrm{Hh}$ signaling microenvironment, induced by androgen deprivation, drives steroidogenesis from benign stromal cell elements within a prostate tumor [29]. In this setting, a Smo antagonist was able to suppress the development of CRPC in a $\mathrm{PCa}$ cell xenograft by reducing endogenous androgen levels in the tumor. Additionally, we previously described tumor cell autonomous activities of Gli mediated by the direct binding of Gli proteins to ARs [30]. Gli proteins recognize the AR N-terminal tau5 transactivation domain (TAD) that regulates ligand-independent activity of AR-FL and is shared by t-ARs [31]. We showed that Gli1 and 2 could co-activate both unliganded and liganded AR-FL as well as t-ARs [30, 31]. Finally, we found that exogenous overexpression of Gli1 or Gli2 was sufficient to drive androgen-independent in vitro growth of androgen growthdependent PCa cells and we attributed this activity to the AR co-activation functions of Gli proteins [30].
We previously localized the AR binding domain on Gli2 to a 270 amino acid region of the Gli2 C-terminus (Gli2aa628-897) [31]. This region shares significant homology with Gli3 (Gli3aa678-936) and it encompasses critical phosphorylation sites that are also targets of $\beta$-TrcP ubiquitination. For Gli3, this region also contains a site of proteolytic cleavage that generates the truncated Gli3repressor (Gli3-R) protein in the absence of Hh signaling.

As a corollary to our studies of the effects of Gli on the transcriptional activity of AR, we explored here the converse idea that $\mathrm{AR}$ affects Gli transcriptional activity in $\mathrm{PCa}$ cells. Our results show that ARs act as a co-activators of Gli-mediated transcription and that transcriptionally-active AR engagement with the protein processing domain (PPD) on Gli3 regulates its proteolytic processing and transcriptional outputs. Furthermore, we show that interference with the intracellular AR-Gli interaction using a decoy peptide can diminish the ability of AR to act as a Gli transcriptional activator.

\section{Results}

\section{Transcriptionally active ARs upregulates Gli transcriptional activity in PCa cells}

Using a panel of five $\mathrm{AR}+\mathrm{PCa}$ cell lines (parental [LNCaP]; androgen growth-independent [LNCap-AI and LN95]), LAPC4 and a CWR1 cell variant, R1-D567, that expresses only a C-terminal truncated AR (AR-e567), we tested whether androgen (R1881) affected expression of a luciferase reporter under the control of a Gli-responsive promoter (Gli-luc). Each of the LNCaP variants and LAPC4 upregulated reporter expression in the presence of R1881 (Fig. 1a). Addition of enzalutamide (Enz), an AR antagonist, blocked this upregulation. While androgen did not affect reporter expression in R1-D567 cells, they had higher overall basal Gli reporter activity compared to cells that express AR-FL. Transfection of a constitutive-active truncated $\mathrm{AR}$ variant, $\mathrm{AR}-\mathrm{V} 7$, into $\mathrm{AR}^{-}$(PC3) or $\mathrm{AR}^{+}$ (LNCaP) $\mathrm{PCa}$ cells also upregulated Gli reporter in the presence or absence of androgen. (Figs. 1b, c). For LNCaP cells, that only express AR-FL, the AR-V7-enhanced reporter activity was not diminished by co-transfection with AR-FL siRNA, in fact, AR-FL knockdown further enhanced the ability of AR-V7 to induce Gli reporter activity (Fig. 1c). For androgen-responsive LNCaP variants, knockdown of AR-FL blocked the upregulation of Glireporter expression induced by androgen (Fig. 1d). We also tested whether androgens affected the expression of endogenous Gli-target genes, Gli1 and Ptch1 in $\mathrm{AR}^{+} \mathrm{PCa}$ cells. Androgen significantly upregulated the expression of Gli1 and Ptch1 at the mRNA and protein levels (Figs. 1e, f) in 

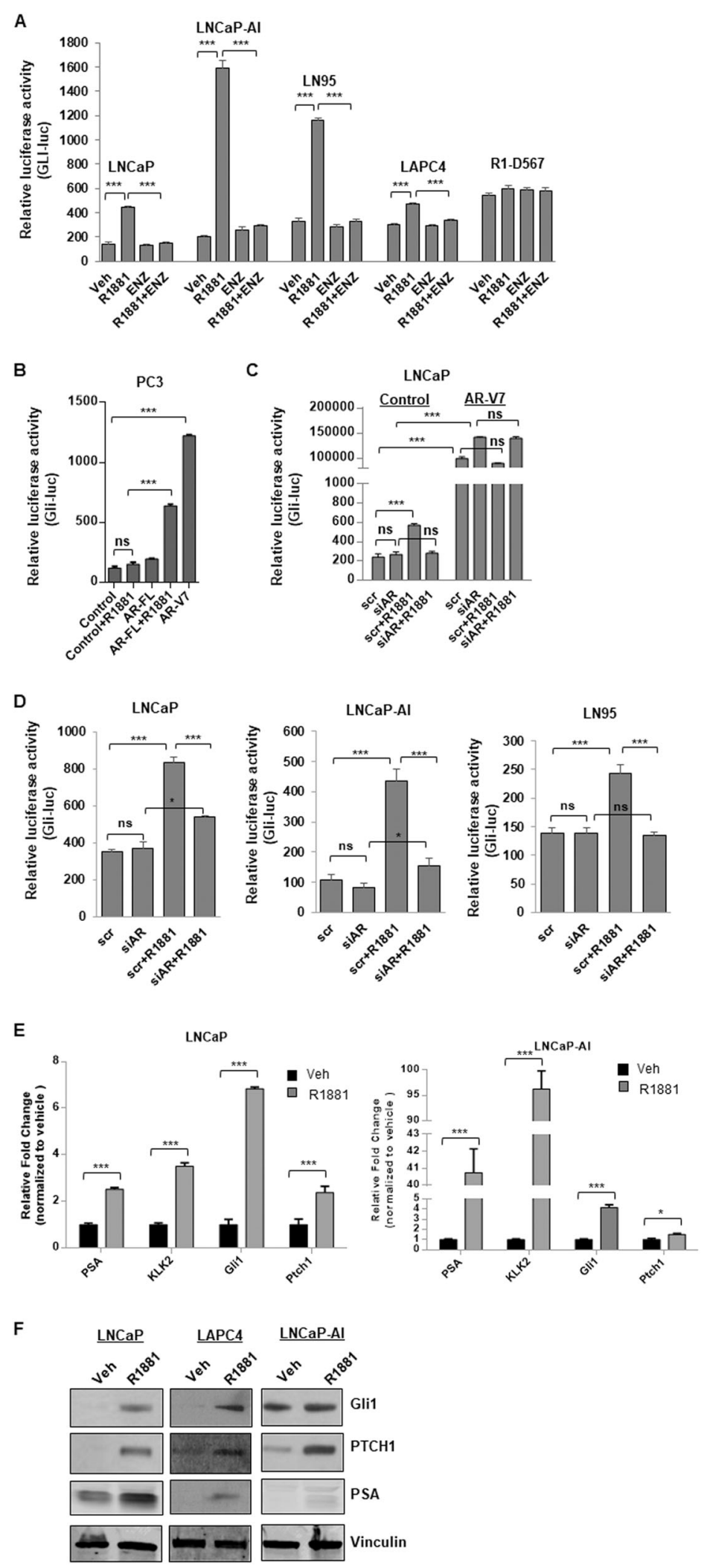
Fig. 1 Transcriptionally-active androgen receptors upregulate Gli transcriptional activity in PCa cells. A panel of AR+ PCa cells were transfected with Gli-luc reporter along with pCMV-eGFP, and (a) cultured in CS-FBS media in the presence of vehicle (Veh), R1881 (1 $\mathrm{nM})$, Enzalutamide (ENZ, $10 \mu \mathrm{M})$ or both; or (b) AR- PC3 cells were co-transfected with Gli-luc reporter and control (empty) vector with AR-FL or ARV7. R1881 was added where indicated. Bars indicate relative luciferase activities. c LNCaP cells were co-transfected with Gli-luc and control vector or AR-V7 vector with a scrambled siRNA (scr) or siRNA targeting AR-FL (siAR). R1881 was added where indicated. Bars indicate relative luciferase activity. d Cells indicated were co-transfected with Gli-luc and control (scr) siRNA or siAR in the presence or absence of R1881, as indicated. a-d Luciferase activities were measured in cell protein extracts and normalized to GFP fluorescence intensities. e LNCaP or LNCaPAI cells were cultured in CS-FBS in the presence or absence of R1881. The mRNA levels of endogenous AR targets (PSA, KLK2) and Gli targets (Gli1, Ptch1) were determined through qPCR and bars indicate the relative fold-change compared to vehicle-treated specimens. a-e Bars represent mean $\pm \mathrm{SD} ; * P<0.05 ; * * * P<0.001 ;$ ns not significant. $\mathbf{f}$ Changes in expression of endogenous Gli target proteins were also confirmed in LNCaP, LAPC4 and LNCaP-AI cells treated with R1881. Cell lysates were prepared for SDS-PAGE, followed by western blot analysis using indicated antibodies

LNCaP and its androgen growth-independent variant LNCaP-AI. LAPC4 cells showed similar upregulation of Gli target proteins (Fig. 1e) on treatment with androgen.

It was of further interest to compare Smo-driven $\mathrm{Hh}$ activity to AR-driven activity, however $\mathrm{LNCaP}$ and other $\mathrm{AR}+\mathrm{PCa}$ cells are unresponsive to Smo agonists. Using Smo-responsive mouse MC3T3-E1 cells instead, we compared Gli-driven luciferase reporter activity following treatment with SAG, a small molecular Smo agonist, to transient transfection with AR-FL or AR-V7. While SAG treatment approximately doubled expression of the Gli reporter, co-transfection with AR-FL (in the presence of R1881) or AR-V7 (in the absence) increased Gli reporter expression by 100 to 200-fold (Supplementary Figure 1).

\section{Androgen promotes the interaction of AR with Gli3 in PCa cells}

In order to characterize the Gli landscape of PCa cells, we surveyed a broad variety of PCa cell lines for the relative expression of Gli1, Gli2, and Gli3. Analysis of mRNA levels by qPCR showed that Gli3 was the predominant Gli homolog expressed in each; often at levels $>100$-fold higher than Gli1 or Gli2 (Supplementary Figure 2). To determine whether androgen affects the intracellular interaction between AR and Gli3, we first evaluated whether androgen influenced the co-immunopreciptation of Gli3 with AR from extracts of LNCaP cells exogenously overexpressing myc-tagged Gli3. Western blots of ARimmunoprecipitates showed that androgen increased the Gli3 content of AR-pulldowns in a dose-dependent manner
(Fig. 2a). We also used the Proximity Ligation procedure that identifies proteins in close contact in situ, to detect and relatively quantify Gli3-AR complexes in $\mathrm{PCa}$ cells. Treatment of LNCaP cells with R1881 significantly increased the number of Gli-AR complexes (Fig. 2b). These complexes were overwhelmingly localized to the nucleus. Addition of Enz significantly reduced the Gli-AR complexes detected in the presence of R1881 (Fig. 2b).

\section{The AR binding site on Gli encompasses critical phosphorylation sites involved in Gli proteolytic processing}

In order to better understand functions of Gli that might be affected by AR binding, we further refined the AR binding site on Gli2 using GST-pulldown in conjunction with sitespecific mutations. Previously we showed that a 270 aa polypeptide from the Gli2 C-terminus (Gli2aa $\left.{ }_{628-897}\right)$ had AR binding activity. Whereas deleting back the $\mathrm{C}$-terminal end of Gli2 $\mathrm{aa}_{628-897}$ to aa836 had little effect on its ability to bind AR-tau5, further deletions back to aa805 or even aa820 resulted in complete loss of binding to AR-tau5 (Fig. 3a). Deletion of the Gli2 $\mathrm{aa}_{628-897} \mathrm{~N}$-terminal region to aa764 had no effect on binding. These results show that a 73aa polypeptide (Gli2 aa $764-836$ ) is sufficient to bind to ARtau5 and further suggest that a 16 peptide region (Gli2aa $\mathrm{a}_{820-836}$ ) is needed for Gli2 binding to AR-tau5. This small region encompasses an arginine, serine-repeat involved in the regulation of Gli2/Gli3 processing (Fig. 3a). Referred to as the PPD [32, 33], it is a target for phosphorylation that is then recognized by the ubiquitin ligase, $\beta$ TrcP [34]. Ubiquitinylation of full-length Gli3 (GliFL) promotes a site-specific cleavage within the C-terminus to generate the Gli repressor (Gli3-R) [33]. Indeed, when these repeats were mutated (S-R-R-S-S $\rightarrow$ S-D-D-S-S), the Gli2 aa $_{628-897}$ polypeptide was no longer able to bind to ARtau5 (Fig. 3a). Moreover, these site-specific mutations, when incorporated into the full C-terminal domain of Gli2 (Gli2-C), blocked the ability of Gli2-C to co-activate AR as determined by its effects on a luciferase reporter from the androgen-responsive PGC promoter (Supplementary Figure 3). Using a GST-pulldown, we further found that $\beta$-TrcP protein competes with the $\mathrm{N}$-terminal domain of AR for in vitro binding to Gli2 aa $764-836$ (Fig. 3b). We also found that increased exogenous expression of $\beta$-TrcP in LNCaP cells displaced AR from Gli3 immunoprecipitates (Fig. 3c).

These findings suggested that binding of AR to Gli3 might affect its ability to be ubiquitinylated and, ultimately, its post-translational proteolytic processing. LNCaP cells grown in androgen-depleted medium for $48 \mathrm{~h}$ have a low ratio of Gli-FL to Gli3-R (Fig. 4a). Treatment of androgen growth-dependent LNCaP cells with increasing doses of 

interaction of AR with Gli3 in PCa cells. a LNCaP cells were cultured in CS-FBS in the presence or absence of $1 \mathrm{nM}$ R1881. Co-IP confirmed the increase of Gli3-FL in AR immuno-precipitates in dosedependent manner. b LNCaP cells were seeded on coverslips in CS-FBS and treated with vehicle (Veh), R1881,

Enzalutamide (Enz), or both. Proximity ligation assay was performed as described in Materials and Methods. Representative images (Top) were taken and processed using a Zeiss $780 \mathrm{M}$ confocal microscope. Photomicrographs are shown at 40X, oil. Scale bar, $20 \mu \mathrm{m}$. PLA signals (Below) groups and counted by two different investigators (JM, ST) from representative fields [vehicle, 8 fields (95cells); R1881, 15 fields (80 cells), $+\mathrm{R} 1881,14$ fields (52 cells)].
Fig. 2 Androgen promotes the were randomly grouped into 4 ENZ, 10 fields ( 141 cells), ENZ $* P<0.05, * * * P<0.001$

A

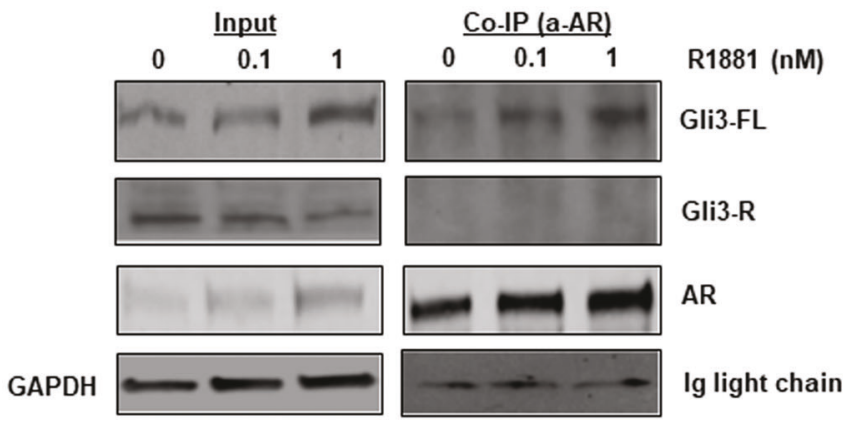

B
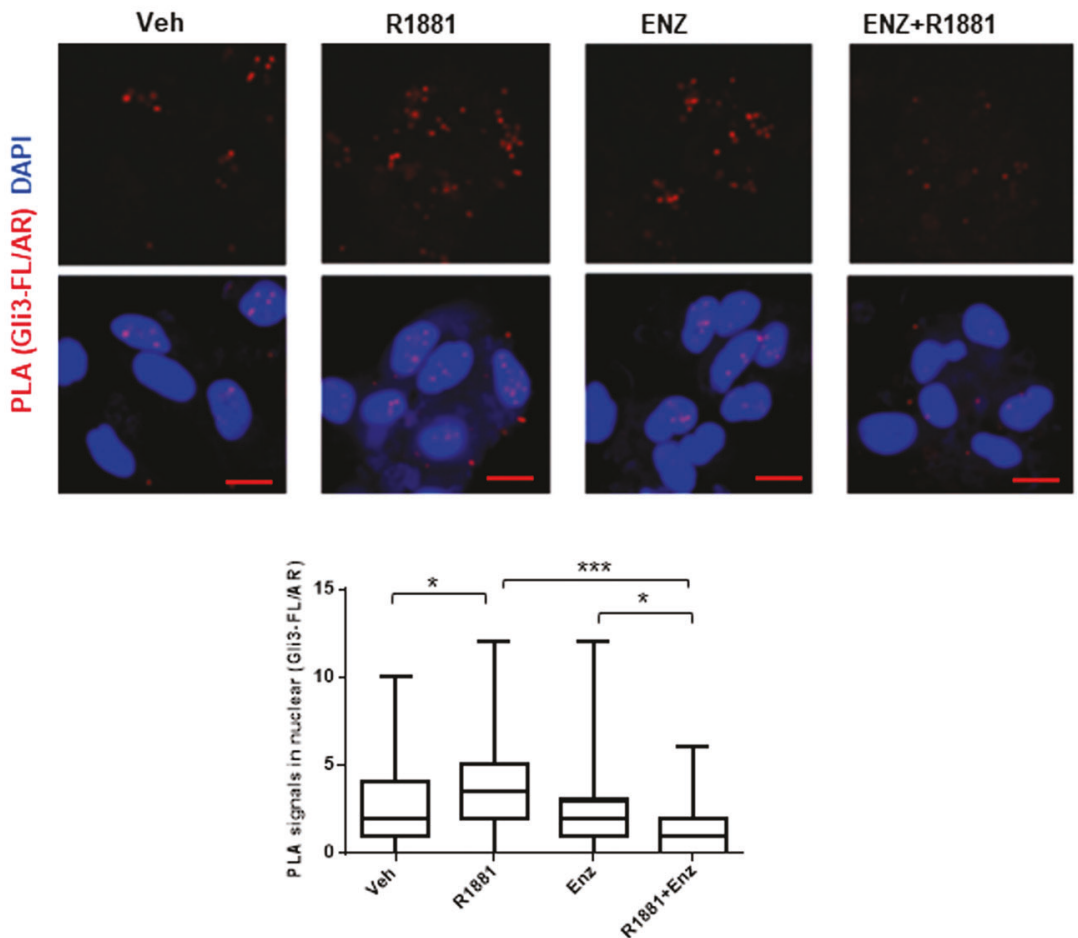

R1881 showed a progressive increase in the ratio of Gli3FL to Gli3R (Fig. 4a, b) (top panel) that corresponded with an increased intensity of Gli-dependent luciferase reporter expression (bottom panel). Androgens increased the Gli3FL/Gli3R ratios in other PCa cell lines (Fig. 4c) whereas knockdown of AR-FL with siRNA reduced Gli-FL/Gli3R ratios. Furthermore, we found that AR knockdown increased the relative degradation rate of Gli-FL (Fig. 4d). Finally, transfection of a t-AR (AR-V7) into LNCaP or PC3 cells also stabilized Gli3-FL (Supplementary Figure 4). It was also notable that LNCaP-AI cells, an androgen growthindependent variant of LNCaP, had a comparatively high endogenous ratio of Gli3-FL/Gli3R (Supplementary Figure 5). Even exogenous myc-tagged Gli3 was differentially processed between LNCaP and LNCaP-AI cells (Supplementary Figure 5).

\section{Decoy peptides from the Gli2 C-terminal domain compete with Gli3 for AR binding and inhibit Hh signaling/Gli activity in PCa cells}

With the AR binding site on Gli2 localized, we tested whether a 270aa truncated polypeptide derived from Gli2, containing the AR binding site, might serve as a decoy that could interfere with the AR-Gli interaction in vivo. This polypeptide $\quad\left(\right.$ Gli2aa $_{628-897}$ or Gli-DP) coimmunoprecipitated with AR following exogenous overexpression in LN95 cells (Fig. 5a) and it displaced myctagged Gli3 from AR co-immunoprecipitates. It also decreased the number of in situ Gli-AR complexes when it was overexpressed in LNCaP-AI cells as was shown by a significant decrease in the Gli3-AR PLA index (Fig. 5b). Finally, Gli-DP reduced expression of the Gli-reporter in LNCaP cells in the presence of R1881 (Fig. 5c) and it 
A

AR binding domain

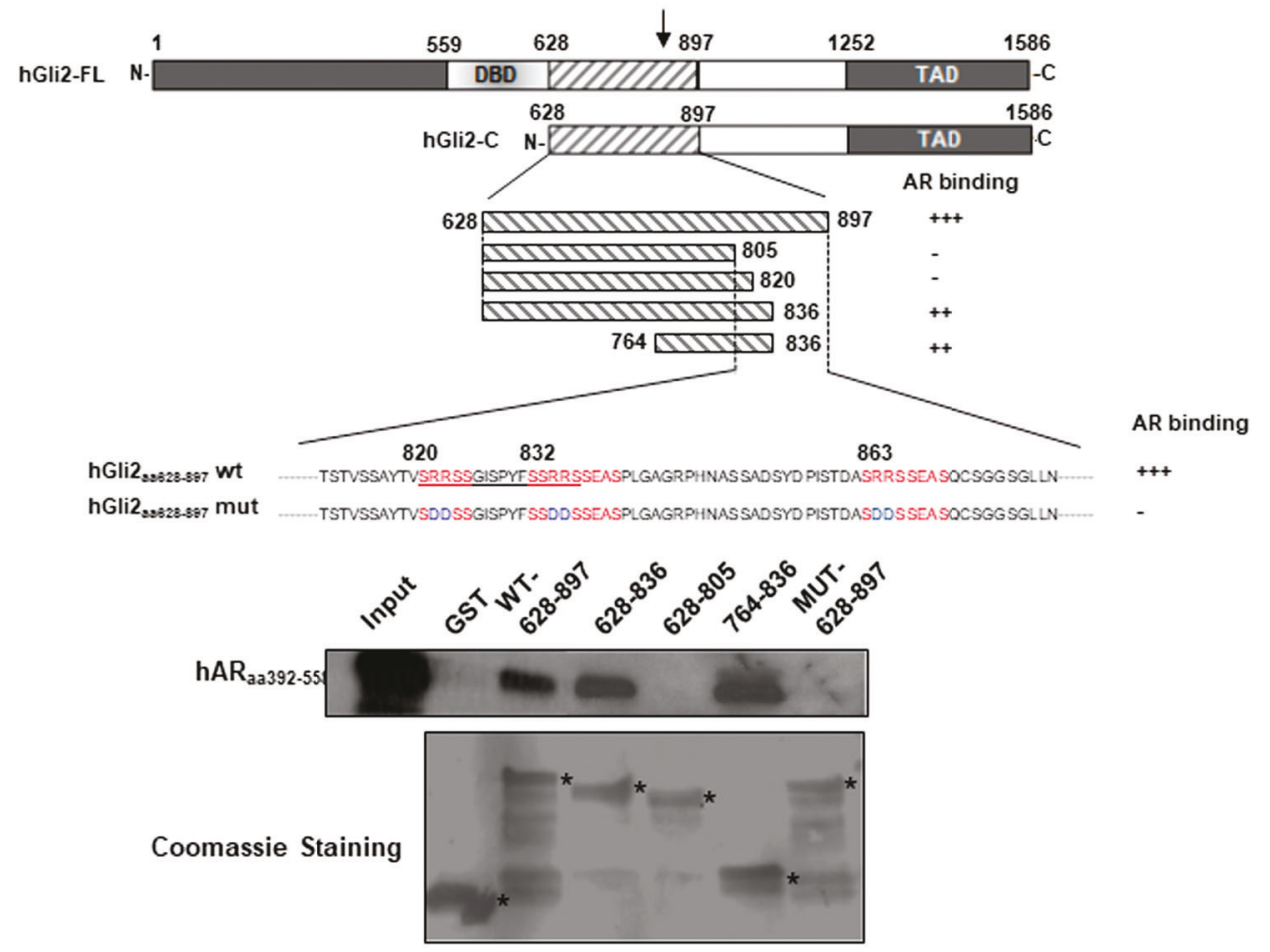

B

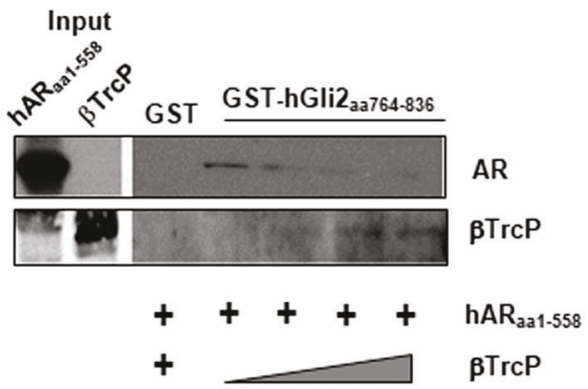

C

293FT

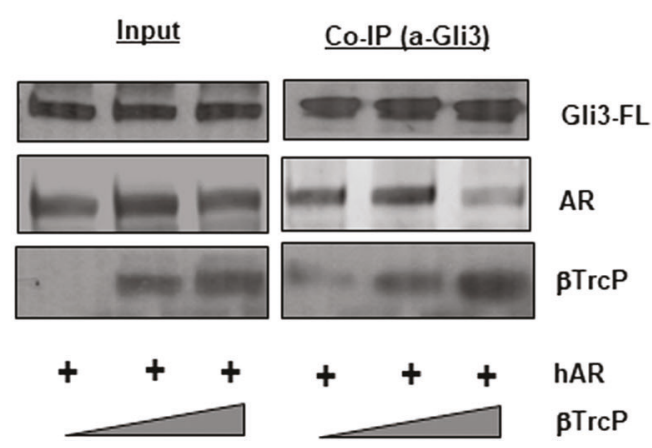


Fig. 3 The AR binding site on Gli2 encompasses phosphorylation and $\beta \operatorname{TrCP}$ binding sites within the C-terminal domain. a (Upper), Schematic diagram of Gli2 protein domains and various sub-fragments (below) tested for binding to AR-tau5 (hARaa ${ }_{392-558}$ ) in GSTpulldowns with binding outcomes indicated on the right. (Middle) Partial sequence of Gli2aa ${ }_{805-897}$ showing phosphorylation site $(-\mathrm{S}-\mathrm{R}-\mathrm{R}-\mathrm{S}-$ repeats [red]) and below, sequence of mutated Gli2aa ${ }_{805-897}$, as was described. (Bottom) Retention of his-tagged ARtau5 polypeptide (Upper) on recombinant GST-tagged G12 polypeptides affixed to glutathione beads confirmed the minimum AR binding domain at Gli2aa $764-836$. Eluates were resolved on SDS-PAGE and western blots were probed for AR-tau5 using an his antibody as described. (Lower) Gli2 recombinant proteins were visualized under Commassie staining (* indicates expected size) after SDS-PAGE. b Competition of $\beta$ TrCP for AR binding site on Gli2. GST-tagged Gli2aa ${ }_{764-836}$ polypeptide was captured on glutathione beads and incubated with $\mathrm{AR} \mathrm{N}$-terminus (hARaa ${ }_{1-558}$ ) (fixed concentration) in the presence of increasing concentration of recombinant $\beta \mathrm{TrCP}$ ( $\beta \operatorname{TrCP} / \mathrm{AR}$ ratio of $0,1 / 4,1 / 2,1 / 1)$. Eluates were separated on SDSPAGE then probed with anti-AR or anti- $\beta \operatorname{TrCP}$ antibody as described. c Extracts of 293FT cells co-transfected with a fixed amount of AR-FL vector and $\beta \operatorname{TrCP}$ (at ratios of $0,1 \mathrm{x}, 2 \mathrm{x} \mathrm{AR}$ ) were immunoprecipitated with anti-Gli3 antibody. Immunoprecipitates were resolved on SDSPAGE and western blots were probed for Gli3-FL (anti-Gli3 antibody), AR (anti-AR antibody) or $\beta \operatorname{TrCP}$ (anti- $\beta \operatorname{TrCP}$ antibody)

suppressed the expression of Gli1 and Ptch1 mRNAs in LNCaP and LNCaP-AI cells (Fig. 5d).

\section{Discussion}

The androgen and Hh signaling pathways are intimately intertwined in PCa cells. Androgens regulate the expression and release of hedgehog proteins from them and androgen deprivation can convert $\mathrm{AR}+\mathrm{PCa}$ cells into virtual hedgehog factories [35, 36]. In turn, hedgehogs released from androgen deprived $\mathrm{PCa}$ cells can stimulate intratumoral steroidogenesis from benign stromal cells in the tumor microenvironment [29, 37], promoting tumor growth after castration. Our continuing research also reveals a unique inter-relationship between the two key transcriptional mediators of these signaling pathways in PCa cells, the AR and the Gli transcription factors. AR is a fundamental regulator of prostate development and an effector of prostate disease states. It is needed for transcription of genes that distinguish the differentiated phenotype of prostate epithelial cells. AR also regulates the growth and invasiveness of PCa cells [38] and, as such, represents a principal therapeutic target for control of metastatic PCa. Glis regulate the expression of genes involved in development, cell growth, cell motility and steroidogenesis and are oncogenic when hyperactive in adult cells/tissues. Clearly, the functional gene networks regulated by these two different transcription systems overlap in $\mathrm{PCa}$ and this is consistent with the long-standing interest in Gli function in PCa cells [39-41].
Gli proteins bind directly to AR [31] and this binding coactivates unliganded as well as liganded AR [30]. Glis recognize the N-terminal AR tau5 TAD [31] that is involved in anomalous unliganded AR-FL activity and this explains the ability of Glis to co-activate t-ARs as well as AR-FL. While we previously suggested that Gli coactivation of AR was an enabler of androgen-independent of PCa cells, our results here shows that AR binding to Gli proteins serves to activate Gli transcription through a noncanonical signaling process in $\mathrm{PCa}$ and other human cells (293FT). Gli is an effector of prostate cancer cell growth [40]. In the future, we need to consider further the extent to which this non-canonical activity contributes to cell growth control in PCa.

For PCa cells that express AR-FL, we found that androgen drives inducible expression of an exogenous Glidependent reporter gene as well as expression of endogenous Gli target genes (Gli1 and Ptch1). This did not occur in a PCa cell line (R1-D567) that is insensitive to androgen because it only expresses a truncated AR variant protein. The presence of Enz, an AR antagonist ligand, or knockdown of AR with siRNA abrogated this response, showing that it was dependent on the presence of transcriptionally active AR. We also found that a constitutively-active t-AR, AR-V7, induced Gli reporter and endogenous target genes when exogenously expressed in both $\mathrm{AR}^{+}$and $\mathrm{AR}^{-} \mathrm{PCa}$ cells. It was notable that knockdown of AR-FL in LNCaP further enhanced the ability of AR-V7 to activate Gli. These responses were recapitulated in Hh-responsive mouse $3 \mathrm{~T} 3$ cells where we found that transfection of full-length or truncated AR provided far more substantial activation of Gli signaling activity than a small molecule Smo agonist. This showed that AR is a relatively robust Gli activator.

Based on an assessment of the relative expression of Gli homologs across a broad panel of PCa cell lines, we concluded that Gli3 is the dominant homolog. Use of a coimmunoprecipitation procedure and proximity ligation assays allowed us to show that androgens quantitatively increased the formation of AR-Gli3 complexes in PCa cells that express AR-FL whereas Enz suppressed Gli-AR complex formation. These outcomes suggest that transcriptionally-active (liganded) AR-FL selectively forms complexes with Gli3 and this is also consistent with the Gli promoting effects of t-AR (in the absence of ligand) that has constitutive transcriptional activity.

While we reported earlier that AR binds to a 270aa polypeptide in the Gli C-terminal domain, further refinement of this binding site on Gli2 (down to a 73aa polypeptide) showed that the serine, arginine-rich repeats within the Gli PPD are necessary for AR binding. Indeed, we found that an AR-tau5 polypeptide competed with $\beta \operatorname{TrCP}$ for binding to Gli2. This finding suggested the possibility that, mechanistically, AR binding to Gli3 protein might 
A

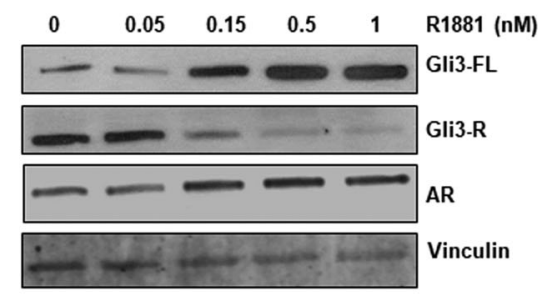

B

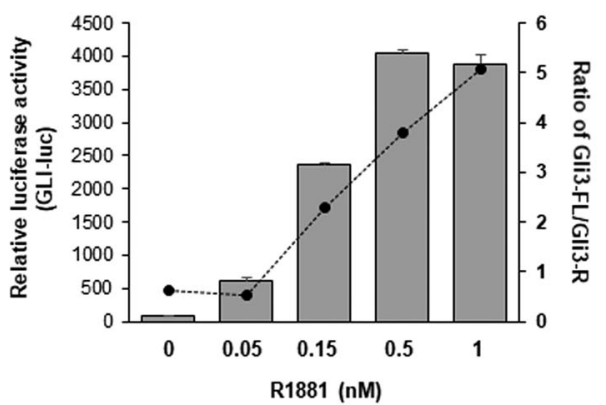

C

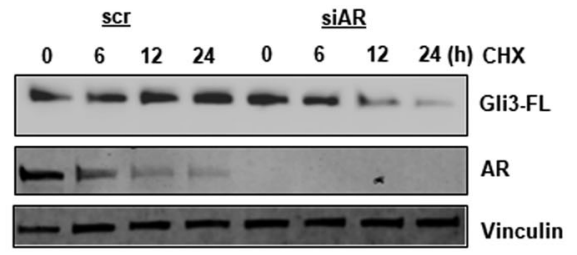

D

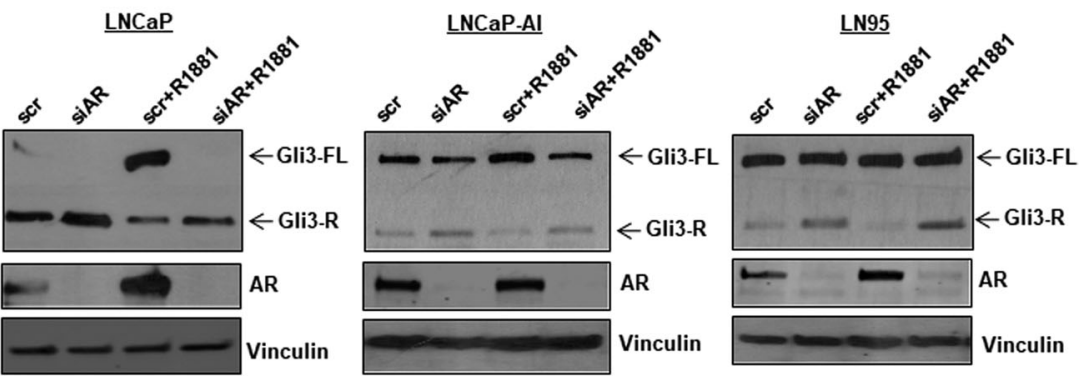

Fig. 4 Transcriptionally active AR stabilizes Gli3-FL. a LNCaP cells were cultured in CS-FBS in the presence of increased doses of R1881, as shown. Western blot analysis confirmed R1881 treatment progressively increased the ratio of Gli3-FL to Gli3R, along with AR protein expression. b In parallel, LNCaP cells stably expressing a Gli luciferase reporter were treated with increased doses of R1881. Luciferase activity was measured and shown in the bar graph. The ratios of Gli3FL to Gli3R were determined by densitometry using Image J software,

mask the PPD from the post-translational modifications and proteolysis involved in Gli3-R generation and stabilize GliFL in its active conformation. This was confirmed by our studies showing that the Gli3-FL/Gli3-R ratio increased in $\mathrm{PCa}$ cells treated with androgens or transfected with a t-AR. Conversely, knockdown of AR reduced Gli-FL/Gli3-R regardless of the presence of androgen and this was consistent with the concomitant loss of Gli signaling. We have, so far, been unable to determine whether AR-FL/t-AR affects Gli2 in our cell models as it is expressed at much lower levels than Gli3 and the anti-Gli2 antibodies we have presented in the dotted line. c Western blot of LNCaP-AI extracts following a cycloheximide chase $(20 \mu \mathrm{g} / \mathrm{ml}$ for hours shown $)$ showed that AR knockdown destabilized Gli3-FL. d Androgen stabilized Gli3FL whereas AR-FL knockdown destabilized Gli3-FL. LNCap, LNCaP-AI or LN95 cells were transfected with scramble (scr) siRNA or siRNA against AR-FL (siAR) and then treated with $1 \mathrm{nM} \mathrm{R} 1881$, as described in Materials and Methods. Cell lysates were resolved on SDS-PAGE and western blot was done with indicated antibodies

tested are inconsistent in detection of this protein by Western blot. But we will continue this effort in the future using $\mathrm{PCa}$ cells that appear to express higher levels of Gli2 (VCaP and LAPC4).

We also tested the idea that a limited Gli2-derived peptide that encompasses the AR binding site might serve as an in vivo decoy to engage AR in PCa cells and displace GliFL. This activity was confirmed by the loss of Gli3 from AR immunoprecipitates in the presence of Gli-DP as well as by a significant decrease of in situ Gli3-AR complexes formed in the presence of Gli-DP. The biological 
A

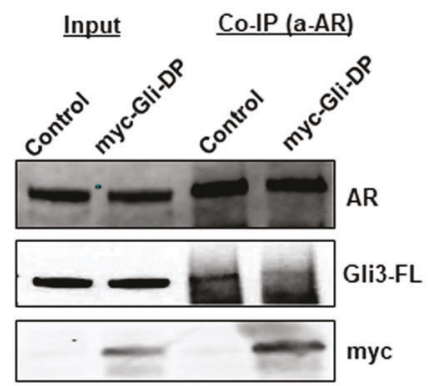

B
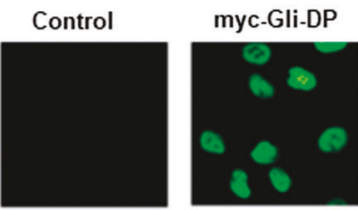

PLA

(Gli3-FL/AR)
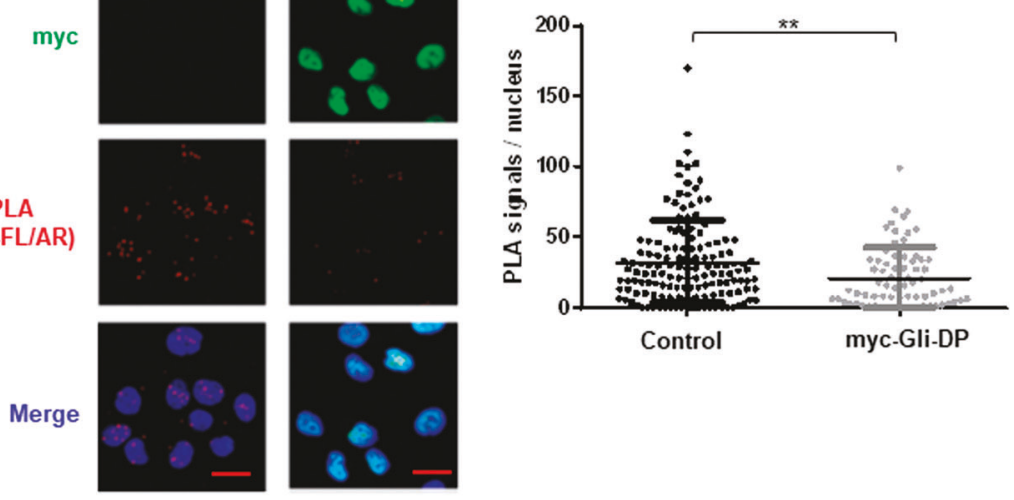

C

D
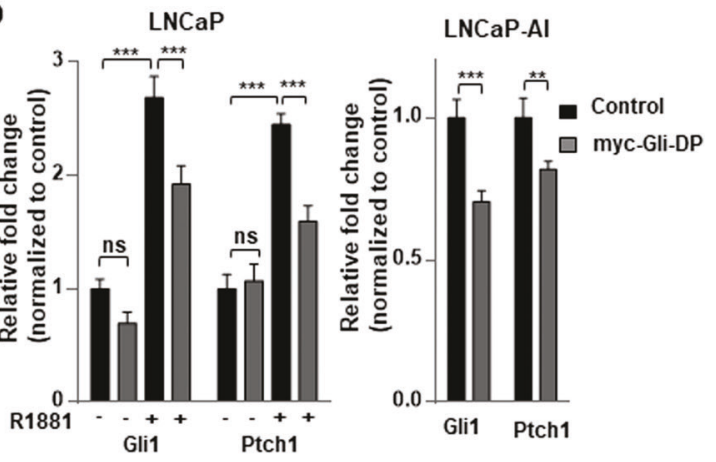

Fig. 5 A decoy peptide (myc-Gli-DP) competes with Gli3 for AR binding and inhibits Gli transcriptional activity in PCa cells. a LN95 cells transiently transfected with control (empty vector) or myc-Gli-DP $\left(\right.$ hGli2 $\left.\mathrm{aa}_{628-897}\right)$ vector. Lysates were immunoprecipitated with antiAR antibody and immunoprecipitates were resolved on SDS-PAGE. Western blots were probed with anti-myc antibody (Gli-DP) or with anti-Gli3 antibody or anti-AR antibody. Results confirmed exogenous myc-Gli-DP interaction with $\mathrm{AR}$ in vivo and its displacement of endogenous Gli3-FL from AR immuno-precipitates. b LNCaP-AI cells overexpressing Dox-inducible myc-Gli-DP or control vector were seeded onto coverslips and cultured in CS-FBS in the presence of 0.2 $\mu \mathrm{g} / \mathrm{ml}$ Dox for $48 \mathrm{~h}$. The samples were then processed for Proximity Ligation Assay as described in Materials and Methods, followed by myc staining to confirm the expression and cellular localization of

myc-Gli-DP. PLA signals were observed under the Zeiss LSM 780 Confocal Laser Microscope and representative images are shown (Left), Objectives, 40x oil. Scale bar, $20 \mu \mathrm{m}$. (Right) PLA quantification was done with the Duolink ${ }^{\circledR}$ ImageTool software.tool,. 147 cells (control) and 72 cells (myc-Gli-DP) were counted. c LNCaP cells were co-transfected with Gli-luc and pCMV-eGFP, along with control $(-)$ and myc-Gli-DP $(+)$ vectors. Cells were further cultured in CS-FBS in the presence or absence of R1881. Protein extracts were assayed for luciferase and normalized to GFP fluorescence intensity. d Endogenous mRNA levels of Gli targets (Gli1 and Ptch1) were determined in Dox-primed LNCaP or -AI cells stably expressing Dox-inducible myc-Gli-DP. Control, Dox-inducible control vector. $n s$ not significant; $* * P<0.01, * * * P<0.001$ 
consequences of this decoy peptide-mediated disruption was a drop in Gli reporter activity as well as decreased expression of endogenous Gli target genes. These observations may have potential clinical relevance for therapeutic development as $\mathrm{Hh} / \mathrm{Gli}$ is known to regulate genes that promote cell proliferation and EMT that is associated with tumor cell invasion and metastasis. Indeed, though not shown here, we do have preliminary evidence that this 270aa Gli2 peptide is an inhibitor of even Enz-resistant PCa cell growth.

There are other questions raised by this finding, especially as to whether other steroid receptors might have similar activities. If so, it could explain the sources of $\mathrm{Hh} /$ Gli activities in other steroid receptor-driven tumors. However, even if this Gli-promoting activity is restricted to AR, it could still be relevant to certain breast, endometrial and ovarian cancers where androgens sometimes have a role in disease establishment or progression. These are also questions that we will be addressing in future work.

\section{Materials and methods}

\section{Cell lines and culture}

Human prostate carcinoma cell lines, LNCaP $(<$ Passage 40, ATCC, Manassas, VA), PC3 (ATCC), LAPC4 (obtained from Dr. Charles Sawyer, MSKCC), and the human embryonic kidney cell line, 293FT (Thermo Fisher, Carlsbad, CA, USA), were maintained in RPMI-1640 or DMEM media supplemented with $10 \%$ FBS (HyClone, Inc., Logan, UT, USA) with streptomycin/penicillin. Androgenindependent LNCaP-AI [30], LN95 (from Dr Stephen Plymate, University of Washington) and CWR1-derived R1-D567 cells (from Dr. Scott Dehm, University of Minnesota) were cultured in RPMI supplemented with $10 \%$ charcoal-stripped FBS (CS-FBS, Hyclone) and streptomycin/penicillin. LNCaP cells were authenticated by short tandem repeat profiling (IDEXX, Inc., Markham, ON, Canada).

\section{Gene constructs and vectors}

AR-FL was provided by Paul Rennie (Vancouver Prostate Centre) and AR-V7 was from Jun Luo (Johns Hopkins Medical Center). The vector expressing Gli3-FL was previously described [31]. Gli luciferase reporter was generated by subcloning an $8 \mathrm{X}$ repeat Gli binding concensus sequence [30] into KpnI and BglII sites of pGL4.48 (Promega, Inc., Madison, WI). A Gli2 decoy peptide was generated by subcloning N-terminal myc-tagged Gli2aa $628-897$ into EcoRI and XbaI sites of pLenti4-TO (Thermo Fisher), followed by an in-frame $\mathrm{C}$-terminal $3 \mathrm{X}$ repeats of a nuclear localization sequence (NLS; forward, 5'-CT AGA GAT CCA AAA AAG AAG AGA AAG GTA GAT CCA AAA AAG AAG AGA AAG GTA GAT CCA AAA AAG AAG AGA AAG GTA TAG T-3', reverse, 5'-CT AGA CTA TAC CTT TCT CTT CTT TTT TGG ATC TAC CTT TCT CTT CTT TTT TGG ATC TAC CTT TCT CTT CTT TTT TGG ATC T-3', inserted into XbaI sites.

\section{Plasmids/siRNA transfection/lentiviral transduction}

For co-immunoprecipitation studies and luciferase reporter assays, above plasmids, as described, were transiently cotransfected into PCa or 293FT cells using lipofectamine 3000 (Thermo Fisher). siRNA targeting AR-FL (AR silencer select, Thermo Fisher, S1539) was transfected into PCa cells using Lipofectamine RNAiMax (Thermo Fisher) by reverse transfection. For generation of doxycyclineinducible LNCaP-AI-myc-Gli2 $628-897$ 3xNLS, two steps of lentiviral transduction were performed as previously described [31]. Briefly, stable LNCaP-AI cells expressing the Tet-Repressor (TR) were generated by transducing lentivirus (pLenti6/TR vector, Thermo Fisher) and ViraPower $^{\mathrm{TM}}$ Lentiviral Packaging Mix (Thermo Fisher). Cells expressing TR were selected in $10 \mu \mathrm{g} / \mathrm{ml}$ Blasticidin and subsequently transduced with lentivirus made from either pLenti4-TO (empty Control) or pLenti4-TO_mycGli2 $628-897 \_3 x N L S$ and were selected under $100 \mu \mathrm{g} / \mathrm{ml}$ Zeocin.

\section{Other recombinant vectors}

Coding sequences of GST-tagged Gli2 fusion-polypeptides, aa628-897, 628-836, 628-805, 764-836 and mutant 628-897 were subcloned into EcoRI and SalI sites of pGEX-5X-3 vector (Clonetech, Mountain View, CA). QuikChange II XL Site-Directed Mutagenesis Kit (Stratagene, La Jolla, CA) was utilized to introduce Aspartate (D) for Arginine (R) residues of the respective G12 phosphorylation sites [32]. Mutagenesis primers used were, amino acid $821 \mathrm{R} / \mathrm{D}$ and $822 \mathrm{R} / \mathrm{D}, 5^{\prime}-\mathrm{C}$ ACC GTG AGC GAC GAC TCC TCC GGC ATC TC-3'; amino acid 833 R/D and 834 R/D, 5'-TAC TTC TCC AGC GAC GAC TCC AGC GAG GCC-3'; amino acid 864 R/D and 865 R/D, 5'-ACG GAC GCG TCG GAC GAC TCG AGC GAG GCC-3'.

\section{Antibodies and reagents}

Mouse anti-human AR (441) antibody (sc-7305) was from Santa Cruz Biotech. (Santa Cruz, CA); rabbit anti-human Gli1 (2553), Ptch1 (2468), PSA (5877), $\beta$-TrCP (4394), and GAPDH (5174) antibodies were from Cell Signaling (Danvers, MA); rabbit anti-human Gli3 antibody (GTX26050) was from GeneTex Inc., Irvine, CA; mouse anti-Vinculin (V9131) from Sigma-Aldrich (Oakville, ON, 
Canada) and mouse anti-myc antibody (05-724) was from EMD Millipore (Etobicoke, ON, Canada). Enz was purchased from Chemexpress Co., Ltd (Shanghai, P.R. China). R1881 was from Perkin Elmer, Shelton, CT and doxycycline was from Sigma, Aldrich and was used at $200 \mathrm{ng} / \mathrm{ml}$ where indicated.

\section{GST pull-down assays}

GST-tagged Gli2 polypeptides were isolated from transfected E. coli BL21 (DE3) pLysS cells (Promega, Inc) using glutathione-sepharose 4B beads (Amersham, Piscataway, $\mathrm{NJ})$ and were visualized after SDS-PAGE with Coomassie blue staining. Human AR truncates (aa1-558, aa392-558) [31] or $\beta \operatorname{TrCP}$ (p4489 Flag- $\beta \operatorname{TrCP}$, Addgene \#10865 [42]) proteins were prepared by Quick Coupled $\mathrm{T} 7 \mathrm{~T}_{\mathrm{N}} \mathrm{T}$ in vitro transcription/translation kit (Promega) in the presence of methionine. These proteins were pre-cleared with GSTbound glutathione beads prior to incubation with GSTtagged recombinant proteins. Immune complexes captured on glutathione beads were eluted for sodium dodecyl sulfate polyacrylamide gel electrophoresis (SDS-PAGE) followed by western blot analysis with appropriate antibodies. For controls, in vitro translated AR truncates and $\beta$-TrCP proteins were incubated with GST beads alone and eluates were analyzed as above.

\section{Western blot and co-immunoprecipitation}

Cell extracts were prepared in 1\% NP-40 lysis buffer as described [31] with $50 \mathrm{mM} \mathrm{Na} \mathrm{F,} 1 \mathrm{mM}$ Orthovanadate, $10 \mathrm{mM}$ Iodoacetamide, $1 \mathrm{mM}$ EDTA, $0.25 \% \mathrm{Na}$ Deoxycholate, $1 \mathrm{mM}$ PMSF, and protease inhibitor cocktail (Roche Diagnostics, Laval, QC, Canada). For Co-Immunoprecipitation, $1.5-3 \mathrm{mg}$ of whole cell lysates were precleared with isotype matching $\operatorname{IgG}$ for $1 \mathrm{~h}$ at room temperature, prior to incubation with 4-6 $\mu$ g (per mg lysates) rabbit anti-Gli3 / mouse anti-AR antibodies overnight at $4{ }^{\circ}$ C. Immune-complexes were precipitated with $30 \mu \mathrm{l} 50 \%$ slurry of Protein A/G-Agarose (Thermo Fisher) for an additional $4 \mathrm{~h}$. For SDS-PAGE, 30-50 $\mu \mathrm{g}$ of cell protein extracts and immunoprecipitates were resolved in pre-cast 4-15\% gradient gels (BioRad, Mississauga, ON, Canada), followed by western blot with appropriate antibodies and detection using ECL (Amersham Biosciences).

\section{Proximity ligation in situ assay (PLA) and confocal microscopy}

PLA was performed with Duolink® In Situ Detection Reagent Fluorescence Kit (Sigma-Aldrich). Briefly, cells were grown on glass coverslips and fixed with 4\% PFA for
$30 \mathrm{~min}$, followed by permeabilization with $0.5 \%$ Triton-X in PBS for 5 min. Cells were blocked with manufacturerprovided blocking solution for $30 \mathrm{~min}$ at $37^{\circ} \mathrm{C}$ and incubated overnight at $4{ }^{\circ} \mathrm{C}$ in a humidified chamber with a mixture of rabbit anti-Gli3 and mouse anti-AR antibodies. Samples were then incubated for $1 \mathrm{~h}$ at $37{ }^{\circ} \mathrm{C}$ with the corresponding PLA probes (anti-rabbit Plus and anti-mouse Minus, provided in kit) conjugated to specific oligonucleotides. The PLA oligonucleotides were hybridized and circularized by ligation $\left(30 \mathrm{~min}\right.$ at $37^{\circ} \mathrm{C}$ ) and amplification $\left(100 \mathrm{~min}\right.$ at $\left.37^{\circ} \mathrm{C}\right)$. The visualization of PLA signals was on a Zeiss LSM 780 Confocal Laser Scanning Microscope (Carl Zeiss, Germany). Quantification of PLA staining was done with Duolink ${ }^{\circledR}$ ImageTool softwear (Sigma-Aldrich).

\section{Quantitative real-time PCR (qPCR)}

Forty-eight or seventy-two hours after siRNA transfection, total RNA was extracted using TRIzol (Thermo Fisher), according to manufacturer's instruction. $1 \mu \mathrm{g}$ of total RNA was used to synthesize cDNA as previously described [31]. Primer sequences employed for PCR amplification are as follows: PSA, forward, 5'-AGT GCG AGA AGC ATT CCC AAC-3'; reverse, 5'-CCA GCA AGA TCA CGC TTT TGT T-3'; KLK2, forward, 5'-GCT GCC CAT TGC CTA AAG AAG-3', reverse, 5'-TGG GAA GCT GTG GCT GAC A-3'; Gli1, forward, 5'-GGC TCG CCA TAG CTA CTG AT-3', reverse, 5'-CCA GCG CCC AGA CAG AG-3'; Ptch1, forward, 5'-CTG GGA GAA GAC GGA GGA GC3', reverse, 5'-CAC AGC TCC TCC ACG TTG GT-3'; 18 s, 5'-TTG ACG GAA GGG CAC CAC CAG-3', reverse, 5'GCA CCA CCA CCC ACG GAA TCG-3'. qPCR was done with SYBR GREEN PCR Master Mix (Roche Diagnostics.) and analyzed in ABI PRISM 7900 HT Fast Real-Time System (Applied Biosystems, Streetsville, ON, Canada). Real-time cycle condition were $2 \mathrm{~min}$ at $50^{\circ} \mathrm{C}$, followed by $10 \mathrm{~min}$ at $95^{\circ} \mathrm{C}$ then 40 cycles of $95^{\circ} \mathrm{C}$ for $15 \mathrm{~s}$ and $60^{\circ} \mathrm{C}$ for $1 \mathrm{~min} .18 \mathrm{~s}$ gene was used as an internal control, and relative fold changes were calculated based on $2^{(-\square \mathrm{CCt})}$ method (http://docs.appliedbiosystems.com/pebiodocs/ 00105622.pdf).

\section{Luciferase reportor assays}

PCa cells were transiently co-transfected with pGL4.28-Gliluc and pCMV-eGFP, along with control or AR-V7 vector. Scrambled siRNA or siRNA against AR-FL was applied $24 \mathrm{~h}$ after plasmids transfection, when used. Luciferase activities were measured using the Dual-Luciferase Reporter Assay System (Promega, Inc.) on the Tecan infinite 200 Pro (Tecan Group Ltd., Männedorf, Switzerland). Values were normalized to relative GFP intensity. 


\section{Statistical analysis}

All values in the figures are presented as mean \pm SD with $n \geq 3$ independent observations. Statistical analyses were performed using Student's $t$ test or Kruskal-Wallis test followed by Dunn's correction with GraphPad Prism (GraphPad Software, Inc. Version 6.0.1, La Jolla, CA). A $P$-value $<0.05$ was considered statistically significant.

Acknowledgements The authors acknowledge the helpful assistance of Dr Mengqian Chen of the South Carolina School of Pharmacy in the generation of the human Gli3 expression vector. Sources of Support: Canadian Institutes of Health Research (CIHR258931); Prostate Cancer Canada-Movember Team T2103; US Department of Defense PCRP W81XWH-10-1-0493; Terry Fox Research Institute New Frontiers PPG 1062.

\section{Compliance with ethical standards}

Conflict of interest All authors declare that they have no competing interests.

Open Access This article is licensed under a Creative Commons Attribution-NonCommercial-ShareAlike 4.0 International License, which permits any non-commercial use, sharing, adaptation, distribution and reproduction in any medium or format, as long as you give appropriate credit to the original author(s) and the source, provide a link to the Creative Commons license, and indicate if changes were made. If you remix, transform, or build upon this article or a part thereof, you must distribute your contributions under the same license as the original. The images or other third party material in this article are included in the article's Creative Commons license, unless indicated otherwise in a credit line to the material. If material is not included in the article's Creative Commons license and your intended use is not permitted by statutory regulation or exceeds the permitted use, you will need to obtain permission directly from the copyright holder. To view a copy of this license, visit http://creativecommons. org/licenses/by-nc-sa/4.0/.

\section{References}

1. Ingham PW, McMahon AP. Hedgehog signaling in animal development: paradigms and principles. Genes Dev. 2001;15:3059-87.

2. Wijgerde M, McMahon JA, Rule M, McMahon AP. A direct requirement for Hedgehog signaling for normal specification of all ventral progenitor domains in the presumptive mammalian spinal cord. Genes Dev. 2002;16:2849-64.

3. Ruiz i Altaba A, Palma V, Dahmane N. Hedgehog-Gli signalling and the growth of the brain. Nat Rev Neurosci. 2002;3:24-33.

4. Xavier GM, Seppala M, Barrell W, Birjandi AA, Geoghegan F, Cobourne MT. Hedgehog receptor function during craniofacial development. Dev Biol. 2016;415:198-215.

5. Tickle $\mathrm{C}$, Barker $\mathrm{H}$. The sonic hedgehog gradient in the developing limb. Wiley Interdiscip Rev Dev Biol. 2013;2:275-90.

6. Finco I, LaPensee CR, Krill KT, Hammer GD. Hedgehog signaling and steroidogenesis. Annu Rev Physiol. 2015;77:105-29.

7. Koebernick K, Pieler T. Gli-type zinc finger proteins as bipotential transducers of Hedgehog signaling. Differentiation. 2002;70:69-76.

8. Jiang J, Hui CC. Hedgehog signaling in development and cancer. Dev Cell. 2008;15:801-12.
9. Hui CC, Angers S. Gli proteins in development and disease. Annu Rev Cell Dev Biol. 2011;27:513-37.

10. Winklmayr M, Schmid C, Laner-Plamberger S, Kaser A, Aberger $\mathrm{F}$, Eichberger $\mathrm{T}$, et al Non-consensus GLI binding sites in hedgehog target gene regulation. BMC Mol Biol. 2010;11:2.

11. Robbins DJ, Fei DL, Riobo NA. The Hedgehog signal transduction network. Sci Signal. 2012;5:re6.

12. Briscoe J, Therond PP. The mechanisms of hedgehog signalling and its roles in development and disease. Nat Rev Mol Cell Biol. 2013;14:416-29.

13. Dai P, Akimaru H, Tanaka Y, Maekawa T, Nakafuku M, Ishii S. Sonic Hedgehog-induced activation of the Gli1 promoter is mediated by GLI3. J Biol Chem. 1999;274:8143-52.

14. Ikram MS, Neill GW, Regl G, Eichberger T, Frischauf AM, Aberger F, et al GLI2 is expressed in normal human epidermis and $\mathrm{BCC}$ and induces GLI1 expression by binding to its promoter. $\mathrm{J}$ Invest Dermatol. 2004;122:1503-9.

15. Agathocleous M, Locker M, Harris WA, Perron M. A general role of hedgehog in the regulation of proliferation. Cell Cycle. 2007;6:156-9.

16. Evangelista M, Tian H, de Sauvage FJ. The hedgehog signaling pathway in cancer. Clin Cancer Res. 2006;12:5924-8.

17. Toftgard R. Hedgehog signalling in cancer. Cell Mol life Sci. 2000;57:1720-31.

18. Rubin LL, de Sauvage FJ. Targeting the hedgehog pathway in cancer. Nat Rev Drug Discov. 2006;5:1026-33.

19. Kasper M, Regl G, Frischauf AM, Aberger F. GLI transcription factors: mediators of oncogenic Hedgehog signalling. Eur $\mathrm{J}$ Cancer. 2006;42:437-45.

20. Yang L, Xie G, Fan Q, Xie J. Activation of the hedgehogsignaling pathway in human cancer and the clinical implications. Oncogene. 2010;29:469-81.

21. Lauth M, Toftgard R. Non-canonical activation of GLI transcription factors: implications for targeted anti-cancer therapy. Cell Cycle. 2007;6:2458-63.

22. Han B, Qu Y, Jin Y, Yu Y, Deng N, Wawrowsky K, et al FOXC1 activates Smoothened-Independent Hedgehog Signaling in Basallike Breast Cancer. Cell Rep. 2015;13:1046-58.

23. Zhou J, Zhu G, Huang J, Li L, Du Y, Gao Y, et al Non-canonical GLI1/2 activation by PI3K/AKT signaling in renal cell carcinoma: A novel potential therapeutic target. Cancer Lett. 2016;370:313-23.

24. Ryan CJ, Tindall DJ. Androgen receptor rediscovered: the new biology and targeting the androgen receptor therapeutically. J Clin Oncol. 2011;29:3651-8.

25. Dehm SM, Tindall DJ. Molecular regulation of androgen action in prostate cancer. J Cell Biochem. 2006;99:333-44.

26. Yuan X, Cai C, Chen S, Chen S, Yu Z, Balk SP. Androgen receptor functions in castration-resistant prostate cancer and mechanisms of resistance to new agents targeting the androgen axis. Oncogene. 2014;33:2815-25.

27. Ware KE, Garcia-Blanco MA, Armstrong AJ, Dehm SM. Biologic and clinical significance of androgen receptor variants in castration resistant prostate cancer. Endocr Relat Cancer. 2014;21: T87-T103.

28. Penning TM. Mechanisms of drug resistance that target the androgen axis in castration resistant prostate cancer (CRPC). J Steroid Biochem Mol Biol. 2015;153:105-13.

29. Lubik AA, Nouri M, Truong S, Ghaffari M, Adomat HH, Corey $\mathrm{E}$, et al Paracrine sonic hedgehog signaling contributes significantly to acquired steroidogenesis in the prostate tumor microenvironment. Int J Cancer. 2017;140:358-69.

30. Chen M, Feuerstein MA, Levina E, Baghel PS, Carkner RD, Tanner MJ, et al Hedgehog/Gli supports androgen signaling in androgen deprived and androgen independent prostate cancer cells. Mol Cancer. 2010;9:89. 
31. Li N, Chen M, Truong S, Yan C, Buttyan R. Determinants of Gli2 co-activation of wildtype and naturally truncated androgen receptors. Prostate. 2014;74:1400-10.

32. Pan Y, Wang B. A novel protein-processing domain in Gli2 and Gli3 differentially blocks complete protein degradation by the proteasome. J Biol Chem. 2007;282:10846-52.

33. Schrader EK, Harstad KG, Holmgren RA, Matouschek A. A three-part signal governs differential processing of Gli1 and Gli3 proteins by the proteasome. J Biol Chem. 2011;286:39051-8.

34. Wang B, Li Y. Evidence for the direct involvement of $\{$ beta $\}$ TrCP in Gli3 protein processing. Proc Natl Acad Sci USA. 2006;103:33-38.

35. Chen M, Tanner M, Levine AC, Levina E, Ohouo P, Buttyan R. Androgenic regulation of hedgehog signaling pathway components in prostate cancer cells. Cell Cycle. 2009;8:149-57.

36. Sirab N, Terry S, Giton F, Caradec J, Chimingqi M, Moutereau S, et al Androgens regulate Hedgehog signalling and proliferation in androgen-dependent prostate cells. Int $\mathrm{J}$ Cancer. 2012;131:1297-306.
37. Levina E, Chen M, Carkner R, Shtutman M, Buttyan R. Paracrine hedgehog increases the steroidogenic potential of prostate stromal cells in a Gli-dependent manner. Prostate. 2012;72:817-24.

38. Zhu ML, Kyprianou N. Role of androgens and the androgen receptor in epithelial-mesenchymal transition and invasion of prostate cancer cells. FASEB J. 2010;24:769-77.

39. Karhadkar SS, Bova GS, Abdallah N, Dhara S, Gardner D, Maitra A, et al Hedgehog signalling in prostate regeneration, neoplasia and metastasis. Nature. 2004;431:707-12.

40. Sanchez P, Hernandez AM, Stecca B, Kahler AJ, DeGueme AM, Barrett $\mathrm{A}$, et al Inhibition of prostate cancer proliferation by interference with SONIC HEDGEHOG-GLI1 signaling. Proc Natl Acad Sci USA. 2004;101:12561-6.

41. Stecca B, Mas C, Ruiz i Altaba A. Interference with HH-GLI signaling inhibits prostate cancer. Trends Mol Med. 2005;11:199-203.

42. Zhou P, Bogacki R, McReynolds L, Howley PM. Harnessing the ubiquitination machinery to target the degradation of specific cellular proteins. Mol Cell. 2000;6:751-6. 